\title{
Corrigendum
}

\section{DNA-conformation is an important determinant of sequence-specific DNA binding by tumor suppressor p53}

Ella Kim, Nils Albrechtsen and Wolfgang Deppert

Oncogene, 1997; 15, 857-869.

Due to a technical mistake, the authors of this paper inadvertently failed to acknowledge the help of Dr Thierry Soussi, Institut Curie, Paris, France, by providing us with his method for the purification of recombinant p53 from baculovirus infected insect cells. 